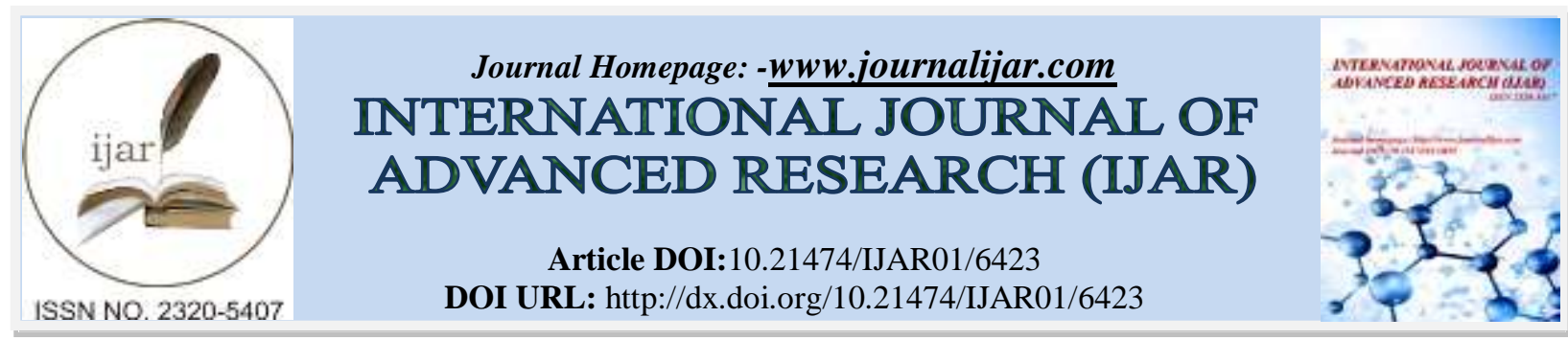

RESEARCH ARTICLE

\title{
RISK FACTORS FOR RETINAL VENOUS OCCLUSIVE DISORDERS IN YOUNGER PATIENTS.
}

\section{"Dr. G. V. Prasad MD ${ }^{1}$ and Dr. Ch. Ramakrishna MBBS ${ }^{2}$.}

1. Civil Surgeon, Department of Ophthalmology, Andhra Medical College, Visakhapatnam.

2. Junior Resident, Department of Ophthalmology, Andhra Medical College, Visakhapatnam.

\section{Manuscript Info}

Manuscript History

Received: 03 December 2017

Final Accepted: 05 January 2018

Published: February 2018

Key words:-

Retinal Venous occlusions, Younger age group, Homocysteine, Hypertension, Hyperlipidemia, Diabetes Mellitus.

\section{Abstract}

Aim: To evaluate the various risk factors in Retinal Venous Occlusive diseases in young patients below 51 years of age.

Materials and Methods: Patients of less than 51 years of age, diagnosed with Retinal venous occlusive disorders (CRVO-Central Retinal Venous Occlusion, HRVO-Hemi retinal Vein Occlusion, BRVO-Branch retinal Vein Occlusion) were investigated for various risk factors . The study was conducted at Retina clinic, Department of Ophthalmology, Government Regional Eye Hospital, Andhra Medical College, Visakhapatnam from august 2017 to January 2018.

Results: A total of 25 patients were recruited for the study. The mean age of the patients in our study was 44 years, the youngest patient was of 25 years and the oldest patient was of 50 years of age. There were 12 males (48\%) and 13 females (52\%) in our study. Elevated Plasma Homocysteine levels were present in 20 patients accounting for $80 \%$ of the cases. Hypertension was present in 16 patients accounting for 64\% of the cases. Hyperlipidemia (Elevated Serum Cholesterol or reduced Serum High Density Lipoproteins (HDL) levels) was present in 13 patients accounting for $52 \%$ of the cases. Diabetes Mellitus was present in 9 patients accounting for $36 \%$ of the cases.

Conclusion: Retinal venous occlusive disorders are one of the common causes of loss of vision. In our study we found that elevated Plasma Homocysteine levels, Hypertension, Hyperlipidemia and Diabetes Mellitus were present in patients of retinal venous occlusive disorders. Hence, investigations must be carried out to detect these conditions in patients diagnosed with retinal venous occlusive disorders. Identifying and treating these conditions can help in preventing recurrences of retinal venous occlusive disorders and can also help in prevention of systemic vaso-occlusive events in future. Primordial prevention in the form of lifestyle modifications in the younger population will also help in preventing occurrence of these risk factors which in turn will prevent the occurrence of ocular as well as systemic vaso-occlusive disorders.

Copy Right, IJAR, 2018,. All rights reserved.

\section{Introduction:-}

Retinal Venous occlusive disorder is the second most common vascular diseases of the retina after diabetic retinopathy typically affecting people of 50 years of age or more. The disease is also seen in people younger than 50 
years of age. Retinal venous occlusions are classified based on the level of obstruction into central or branch retinal venous occlusions. Various ocular and systemic risk factors have been found to be associated with retinal venous occlusive disorders. The various risk factors are:

Ocular Risk Factors:

Glaucoma, Trauma, Retinal Vasculitis, Central Retinal Artery Occlusion, Drusen, Papilloedema, Arterio-venous malformations.

\section{Systemic Risk Factors:}

Age, Hypertension, Hyperlipidemia, Diabetes Mellitus, Oral contraceptive Pill usage, smoking, Thrombophilias (Hyperhomocystinemia, Antiphospholipid antibody syndrome, Factor V Leiden Mutation), Myeloproliferative disorders, Dehydration, Chronic Renal Failure

The purpose of this study was to evaluate the various systemic risk factors for retinal venous occlusive disorders in younger patients below the age of 51 years.

\section{Materials and Methods:-}

The study was conducted at Retina Clinic, Department of Ophthalmology, Government Regional Eye Hospital, Andhra Medical College, Visakhapatnam from August 2017 to January 2018.

\section{Inclusion Criteria:}

All the patients with age $<=50$ years and diagnosed with any type of retinal venous occlusion (CRVO-Central Retinal Vein Occlusion, HRVO-Hemi retinal Vein Occlusion, BRVO-Branch retinal Vein occlusion, Macular vein Occlusion, Tributary Vein Occlusion) were included in the study.

\section{Exclusion Criteria:}

Retinal Venous occlusions diagnosed in people above the age of 50 years were excluded from the study.

Patients satisfying the inclusion criteria were recruited for the study. Informed consent was taken from every participant. Identification data like Name, Age, Sex, Address, and Occupation were collected. A detailed history pertaining to the ocular complaint and history of any associated systemic illnesses was recorded. Best corrected Visual acuity of the patient was recorded in both the eyes using Snellen's Chart. A detailed Ocular Examination was done which included Slit lamp Examination of the Anterior Segment followed by Examination of the Posterior Segment with 78D lens and Slit Lamp Biomicroscopy as well as Indirect Ophthalmoscopy. Intraocular pressure (IOP) was measured with Goldmann'sApplanation Tonometer prior to pupillary dilatation. A thorough Systemic Examination was done which included recording of the vital data-Pulse Rate and Blood Pressure, followed by auscultation of Cardiovascular and Respiratory systems followed by palpation of abdomen for any Organomegaly. A diagnosis of retinal venous occlusion was made based on clinical examination of Fundus with 78D lens with slit lamp and Indirect Ophthalmoscopy.

All the participants diagnosed with retinal venous occlusion were subjected to the following investigations:

- $\quad$ Serum Lipid Profile

- $\quad$ Fasting and Post-Prandial Blood Glucose levels

- $\quad$ Plasma Homocysteine Levels.

- Hypertension was defined as Blood Pressure of $>130 / 80 \mathrm{~mm}$ of $\mathrm{Hg}$ recorded at two different instances with a minimum interval of $6 \mathrm{hrs}$ in between the two recordings, with a manual Sphygmomanometer at the left arm in supine position, if the patient was not a diagnosed case of Hypertension.

- Diabetes Mellitus was defined as Fasting Blood Glucose Level > $125 \mathrm{mg} / \mathrm{dl}$ and/or Post-Prandial Blood Glucose Level $>200 \mathrm{mg} / \mathrm{dl}$ if the patient was not a diagnosed case of Diabetes Mellitus.

- Hyperhomocysteinemia was defined as Plasma Homocysteine Levels $>12 \mu \mathrm{mol} / \mathrm{lit}$

- Hyperlipidemia was defined as Serum Total Cholesterol $>200 \mathrm{mg} / \mathrm{dl}$ or Serum High Density Lipoproteins(HDL) $<40 \mathrm{mg} / \mathrm{dl}$

\section{Statistical Analysis:}

Data was analyzed with the help of Descriptive Statistics. 


\section{Results:-}

In our study, out of the 25 participants, there were 12 males (48\%) and 13 females (52\%)(Figure:1). The mean age of the participants in the study was 44 years, the youngest being 25 years and the oldest being 50 years. There were 18 cases of BRVO (72\%), 6 cases of CRVO (24\%) and 1 case of HRVO (4\%) (Figure:2).

Of the various systemic risk factors studied for association with retinal Venous Occlusions, Hyperhomocystinemia was found in the majority of the patients. 20 cases out of 25 had elevated Plasma Homocysteine levels accounting for $80 \%$ of the study population. The second most common risk factor in our study was found to be Systemic Hypertension. Systemic Hypertension was present in 16 participants accounting for $64 \%$ of the cases. The mean Systolic Blood Pressure was $133.76 \mathrm{~mm}$ of $\mathrm{Hg}$ and the mean Diastolic Blood Pressure was $87.04 \mathrm{~mm}$ of $\mathrm{Hg}$. Hyperlipidemia was the third most common risk factor found to be associated with Retinal Venous occlusion in our study. 13 participants had Hyperlipidemia accounting for 52\% of the cases. 10 patients had elevated Serum Total cholesterol and 3 patients had reduced Serum HDL cholesterol. Diabetes Mellitus was the fourth common risk factor in our study. 9 participants suffered from Diabetes mellitus accounting for $36 \%$ of the study population (Figure:3)(Table-1).

\section{Sex Distribution(Figure:1)}

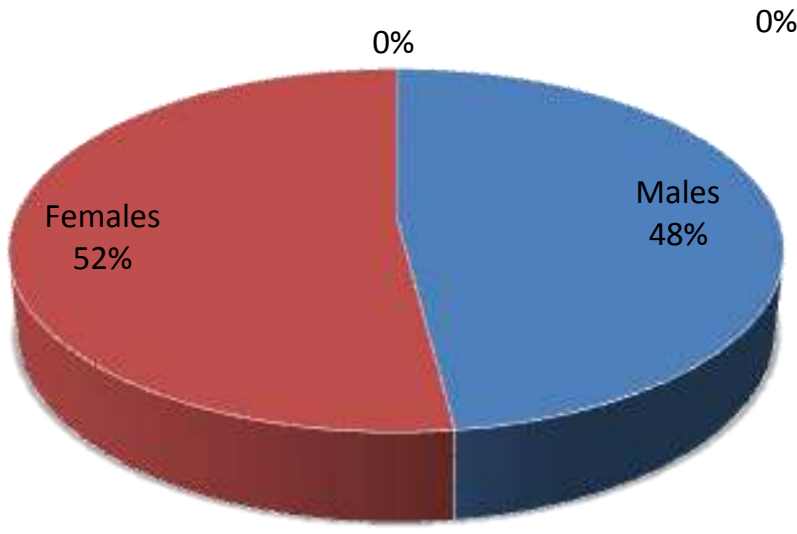

\section{Type of Venous Occlsuion(Figure:2)}

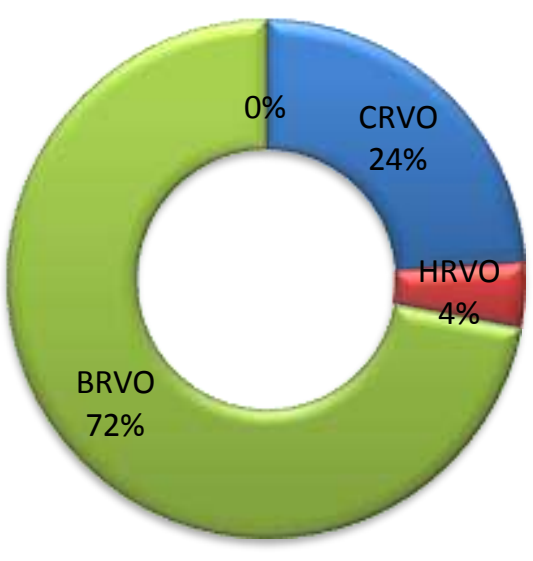




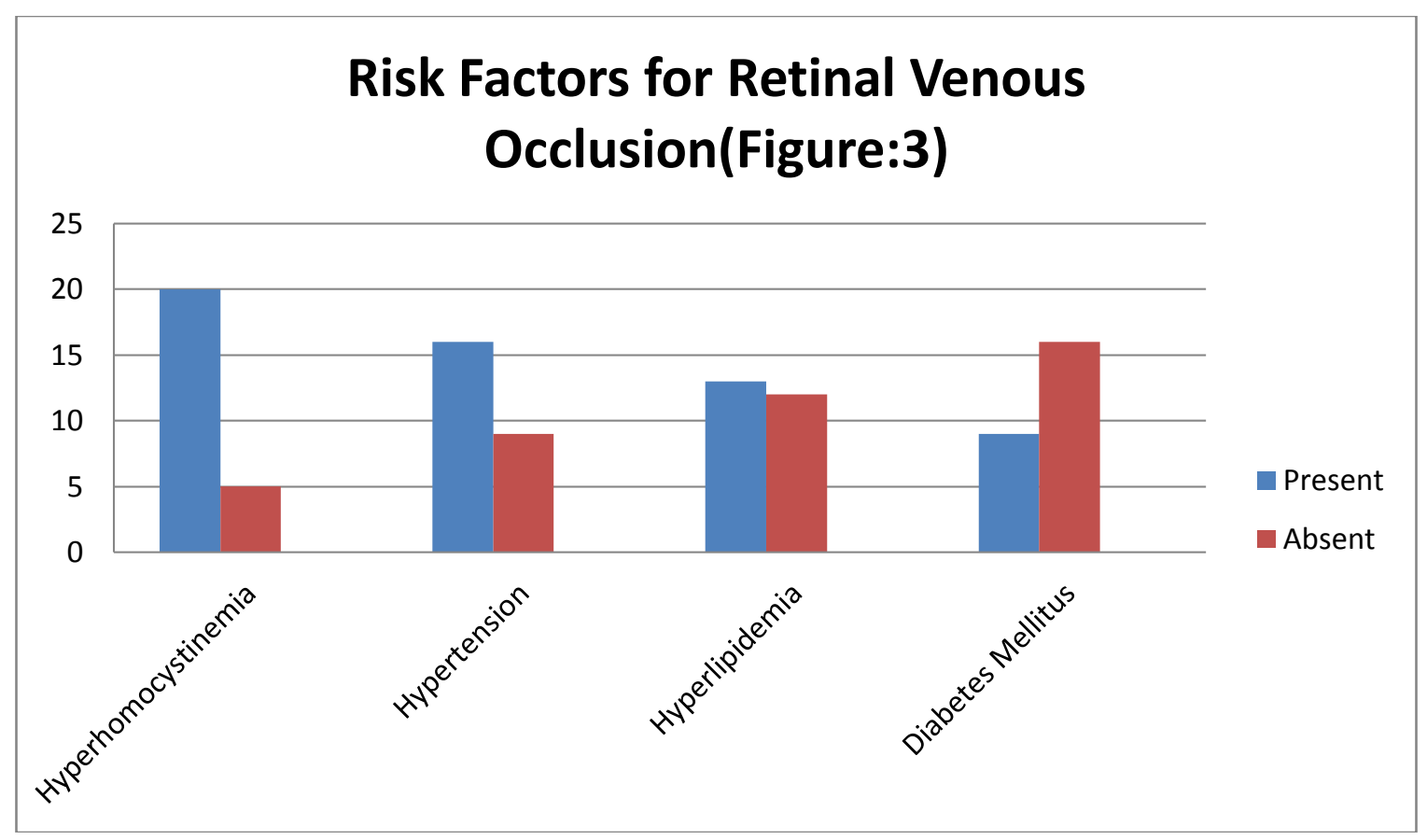

Table-1:

\begin{tabular}{|l|l|}
\hline Risk Factors for Retinal Venous Occlusion & No.of patients with Risk Factors out of 25 participants \\
\hline Hyperhomocystinemia & $20(80 \%)$ \\
\hline Hypertension & $16(64 \%)$ \\
\hline Hyperlipidemia & $13(52 \%)$ \\
\hline Diabetes Mellitus & $09(36 \%)$ \\
\hline
\end{tabular}

\section{Discussion:-}

Our study evaluated 25 patients of $<=50$ years of age diagnosed with retinal venous occlusions for various systemic risk factors associated with Retinal venous occlusive disorders. In our study, Hyperhomocystinemia (80\%) was the most common risk factor associated with Retinal Venous Occlusions followed by Hypertension (64\%), Hyperlipidemia (52\%) and Diabetes Mellitus (36\%) in the decreasing order.

In a study titled "Retinal Vein Occlusion-Risk Factors in Younger Patients" conducted at Government Medical College Kozhikode, they concluded that, detailed evaluation for systemic risk factors should be made mandatory in all cases of retinal vein occlusion. Many of the patients belonged to older age group and Hyperlipidemia was the major single modifiable risk factor among these. It should be kept in mind that in younger population, conventional metabolic risk factors may not be evident in the early stages and hence less common factors like high serum levels of Homocysteine should be looked for and prompt treatment initiated at the earliest. Strict follow up of these patients for evaluation of any systemic diseases in the future should also be done. ${ }^{1}$

In another study conducted by Rajini sharma1 and MohdAyaz Bhat 2 at Department of Ophthalmology, Government Medical College, Jammu titled "Risk Factors in retinal Vein Occlusion", they concluded that Hypertension and Hyperlipidemia were strongly associated with RVO (Retinal venous Occlusion). ${ }^{2}$

In another study titled "Homocysteine in retinal Vascular Occlusions" it was concluded that: The role of Homocysteine in CRVO is complex. Observational studies have shown that CRVO in general population is associated with raised plasma Homocysteine concentration. ${ }^{3}$

Another population based study by Chua B1, Kifley A, Wong TY, Mitchell P, showed that elevated serum Homocysteine is associated with the presence of RVO, independent of other risk factors. ${ }^{4}$ 


\section{Conclusion:-}

Our study shows that Hyperhomocystinemia, Systemic Hypertension, Hyperlipidemia and Diabetes mellitus were significant risk factors associated with Retinal Venous Occlusive Disorders in the younger patients below the age of 51 years. Hence, investigating for these systemic risk factors and taking appropriate corrective measures for these detected abnormalities in young patients diagnosed with retinal venous occlusive disorders will help in preventing the occurrence of future ocular as well as systemic vaso-occlusive events and thereby help in reducing morbidity and mortality. Hypertension, Hyperlipidemia and Diabetes Mellitus are lifestyle disorders which can be prevented by making modifications in our lifestyle as a form of primordial prevention. Hence emphasizing the role of lifestyle modifications on the younger population will definitely help in prevention of occurrence of ocular as well as systemic vaso-occlusive disorders.

\section{References:-}

1. Arun Kumar Ayoor et al, Retinal Vein Occlusion -Risk Factors in Younger Patients JMSCR Volume 05 Issue 10 October 2017;29256-29263.

2. Rajini Sharma, MohdAyazBhat. Risk factors in retinal vein occlusion. International Journal of Contemporary Medical Research 2016;3(4):979-981.

3. Dr. Tirupati Nath1, Dr.Rachit Agrawa12, Dr.Gunjan Prakash3, Homocysteine in Retinal Vascular OcclusionsInternational Journal of Science and Research (IJSR),Volume 5 Issue 1, January 2016;1949-1951.

4. Chua B1, Kifley A, Wong TY, Mitchell P.Homocysteine and retinal vein occlusion: a population-based study.Am J Ophthalmol. 2005 Jan;139(1):181-2. 